\title{
Mutated lamin A modulates stiffness in muscle cells
}

\author{
Maria Chatzifrangkeskou ${ }^{1 *}$, Delf $\mathrm{Kah}^{2 *}$, Janina R. Lange ${ }^{2}$, \\ Wolfgang H. Goldmann ${ }^{2 \#}$, Antoine Muchir ${ }^{1}$
}

\author{
${ }^{1}$ Center of Research in Myology, UPMC-Inserm UMR974, Institut de \\ Myologie, G.H. Pitie Salpêtrière, F-75651 Paris Cedex 13, France \\ ${ }^{2}$ Department of Physics, Biophysics Group, Friedrich-Alexander-University Erlangen- \\ Nuremberg, D-91052 Erlangen, Germany \\ \#Correspondence author: wolfgang.goldmann@fau.de \\ *These authors contributed equally
}

\begin{abstract}
The cytoskeleton is a complex network interlinking filaments that extend throughout the cytoplasm from the nucleus to the plasma membrane. Three major types of filaments are found in the cytoskeleton: actin filaments, microtubules, and intermediate filaments. They play a key role in the ability of cells to both resist mechanical stress and generate force. However, the precise involvement of intermediate filament proteins in these processes remains unclear. Here, we focused on nuclear A-type lamins, which are connected to the cytoskeleton via the Linker

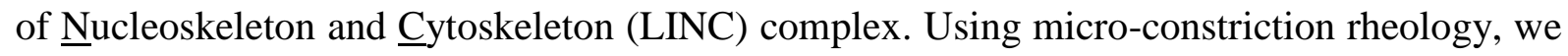
investigated the impact of A-type lamins (p.H222P) mutation on the mechanical properties of muscle cells. We demonstrate that the expression of point mutation of lamin A in muscle cells increases cellular stiffness compared with cells expressing wild type lamin A and that the chemical agent selumetinib, an inhibitor of the ERK1/2 signaling, reversed the mechanical alterations in mutated cells. These results highlight the interplay between A-type lamins and mechano-signaling, which are supported by cell biology measurements.
\end{abstract}




\section{Keywords}

A-type lamin (LMNA); cell nucleus; ERK1/2 signaling; chemical substance selumetinib; cell mechanical properties; micro-constriction rheology

\section{Introduction}

The cytoskeleton is a complex network of microfilaments, microtubules, and intermediate filaments (IFs) that plays a pivotal role in sensing and transmitting both mechanical and chemical stimuli in cells. The cytoskeleton provides functional and structural organization and influences the mechanical properties of cells. Further, it controls many dynamic processes, including cellular stiffness, tractions, and adhesion. So far, most cell mechanical studies focused on elucidating the role of actin filaments and microtubules; however, recent studies described the influence of IF proteins in cellular mechano-signaling, -sensing, and -transduction [1-3]. How IFs function in cells and influence mechanical properties remains open and is presently the focus of much research.

The nuclear lamina is a meshwork of IF proteins that lies beneath the inner nuclear membrane $[4,5]$. One of its principal functions is to control the physical properties of the

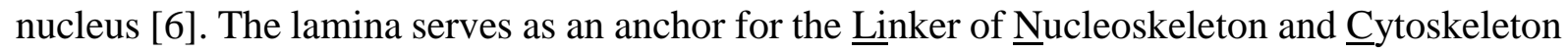
(LINC) complex, which binds the nucleus to the cytoskeleton to control nuclear shape and mechanochemical signaling [7-9]. It is known that the nuclear lamina confers mechanical stability to the nucleus and plays a fundamental role in cellular nuclear function such as nuclear localization, cell migration, chromatin organization, and DNA replication as well as repair [1012]. A-type nuclear lamins have been described to promote nuclear stiffness [13] and are developmentally regulated with their expression proportional to tissue stiffness [14].

Mutations in LMNA, which encode A-type nuclear lamin [15], are responsible for 
autosomal forms of Emery-Dreifuss Muscular Dystrophy (EDMD) [16], a muscular disorder characterized by progressive muscle weakness and wasting associated with early contractures and dilated cardiomyopathy [17]. These mutations further cause limb girdle muscular dystrophy [18], congenital muscular dystrophy [19], or isolated cardiomyopathy without skeletal muscle involvement [20], expanding the phenotypic spectrum of striated muscle disease linked to mutant A-type lamins. So far, the pathological mechanism of these striated muscle diseases remains elusive. To date, the main challenge is how mutations associated to ubiquitously expressed A-type lamin proteins, give rise to pathologies that affect only mechanically stressed muscles [21]. To partially explain the striated muscle diseases, it has been suggested that patients experience mechanical weakness in cells, caused by a disturbed nuclear architecture [22]. This mechanical stress hypothesis could explain the loss of muscle tissue.

We previously demonstrated that the stress-activated extracellular signal-regulated kinase (ERK) 1/2 is hyper-active in EDMD [23]. In striated muscles, active phosphorylated ERK1/2 participates in actin filament disassembly [24] and in the regulation of actin bundling [25]. The actin cytoskeleton controls many dynamic processes, including nuclear and cellular stiffness, cellular tractions, and adhesion. Thus, how nuclear A-type lamins function in cells and influence mechanical properties, remains open and is presently the focus of much research. Previously, Maniotis et al. [10] showed that extracellular mechanical forces can be transmitted to the nucleus of cells, and Isermann and Lammerding [26] reported that the disruption of the connection between the nucleus and the cytoskeleton can lead to striated muscle disease.

We therefore hypothesize that ERK1/2 signaling is related to changes in the mechanical properties of muscle cells expressing mutated A-type lamins and test the chemical inhibitor selumetinib on ERK1/2 activity using micro-constriction rheometry. 


\section{Materials and methods}

\section{Cell culture and reagents}

The generation of stable $\mathrm{C} 2 \mathrm{C} 12$ cells expressing wild type (C2-WT) and H222P (C2-H222P) lamin A has been previously described by [27]. C2-WT and C2-H222P cells were transiently transfected with plasmids, using Lipofectamine 2000 (Invitrogen) according to manufacturer's instructions. A concentration of $50 \mu \mathrm{M}$ selumetinib (AZD6244) was prepared from a stock solution diluted in 5\% dimethylsulfoxyde (DMSO). Cells were cultured in skeletal muscle cell growth medium supplemented with a 5\% PromoCell supplement Mix (PromoCell), 1.5\% Glutamax (Gibco), 0.3\% Gentamycin (Gibco), and 10\% FCS. These cells were then incubated with cytochalasin D for 45 min and selumetinib for $15 \mathrm{~h}$.

\subsection{Wound healing assay}

For wound healing assays, equal numbers of C2-WT and C2-H222P cells were plated at high density in 6-well plates and grown to confluency overnight. The next day, the monolayer was scratched using a $10 \mu \mathrm{L}$ pipette tip, and the media was changed to remove debris, and the wound was imaged over time. For the wound healing assay using selumetinib, $\mathrm{C} 2-\mathrm{H} 222 \mathrm{P}$ cells were treated with $50 \mu \mathrm{M}$ selumetinib for $15 \mathrm{~h}$ prior to wounding.

\subsection{Protein extraction and immunoblotting}

Total proteins were prepared by resuspending cultured cells in extraction buffer (Cell Signaling) with the addition of protease inhibitors including $25 \mu \mathrm{g} / \mathrm{ml}$ aprotinin, $10 \mu \mathrm{g} / \mathrm{ml}$ leupeptin, $1 \mathrm{mM}$ 4-[2-aminoethyl]-benzene sulfonylfluoride hydrochloride, and $2 \mathrm{mM} \mathrm{Na}_{3} \mathrm{VO}_{4}$. The lysates were sonicated at 3 pulses of $10 \mathrm{~s}$ and $30 \%$ amplitude to allow dissociation of proteins from chromatin and solubilization. Sample protein content was determined by the 
Bicinchoninic Acid Assay protein assay (Thermo Fisher Scientific). Extracts were analyzed by SDS-PAGE using a 10\% gel and transferred onto nitrocellulose membranes (Invitrogen). Subsequent to being washed with Tris-buffered saline containing 1\% Tween 20 (TBS-T), the membranes were blocked by 5\% bovine serum albumin (BSA) in TBS-T for $1 \mathrm{~h}$ at room temperature, then incubated with the appropriate antibody overnight at $4{ }^{\circ} \mathrm{C}$. Further, to being washed with TBS-T, the membranes were incubated with horseradish peroxidase-conjugated anti-rabbit or anti-mouse antibodies for $1 \mathrm{~h}$ at room temperature. After washing with TBS-T, the signal was shown, using Immobilon Western Chemiluminescent Horse Radish Peroxidase (HRP) Substrate (Millipore) on a G-Box system with Gene Snap software (Ozyme).

\subsection{Antibodies}

The following primary antibodies were used: anti-cortactin (Cell Signaling), antiphosphorylated (S405) cortactin, and anti-phosphorylated (S418) cortactin (both a gift from S. A. Weed), anti-phosphorylated FAK, anti-paxillin, anti- $\alpha$ actinin, anti-zyxin, antiphosphorylated ERK1/2 antibody (Cell Signaling), and anti-ERK1/2 (Santa Cruz Biotechnology). Secondary antibodies for immuno-fluorescence were: Alexa Fluor-488 conjugated goat anti-rabbit IgG, Alexa Fluor 568-conjugated goat anti-mouse IgG, and AlexaFluor-488-conjugated donkey anti-goat IgG (Life Technologies). Secondary antibodies for immunoblotting were HRP-conjugated rabbit anti-mouse and goat-anti rabbit IgG (Jackson ImmunoResearch).

\subsection{Immunofluorescence microscopy}

C2C12 cells were grown on coverslips, washed with PBS, and fixed with $4 \%$ paraformaldehyde in PBS for 10 min. Cells were permeabilized with $0.2 \%$ Triton X-100 diluted in PBS for 7 min, and non-specific signals were blocked with $0.2 \%$ Triton X-100 and 5\% BSA for $30 \mathrm{~min}$. The 
samples were then incubated with a primary antibody for $1 \mathrm{~h}$ in PBS with $0.1 \%$ Triton X-100 and $1 \% \mathrm{BSA}$ at room temperature. Cells were washed with PBS and incubated for $1 \mathrm{~h}$ with a secondary antibody. Cells and slides were then mounted in Vectashield mounting medium with DAPI (Vector Laboratories). Immunofluorescence microscopy was performed using an Axiophot microscope (Carl Zeiss). All images were processed using Adobe Photoshop 6.0 (Adobe Systems).

\subsection{Micro-constriction rheology}

The micro-constriction setup and evaluation of quantitative cell mechanical properties have been described in detail by [28]. In brief, the microfluidic device consists of eight parallel constrictions connected to a single inlet and outlet with a low-resistance, pressure-equalizing bypass. The suspended cells first passed through a filter mesh before the flow was divided into eight parallel constriction branches. In each branch, the cells were squeezed through a micronsized constriction and had to conform to the size of the constriction in order to pass through. The width and height of the device was in the range of the cell diameter constriction: width of $7.7 \mu \mathrm{m}$ and height of $18.9 \mu \mathrm{m}$ for C2-WT and C2-H222P cells with/without selumetinib. With these dimensions, an average cell entry time between 5 and $1000 \mathrm{~ms}$ can be achieved. The cell transit was continuously monitored by a high-speed camera (GE680, Allied Vision, Germany; 750fps) in bright-field mode on an inverted microscope (DM-IL, Leica) with 10x magnification with a custom-written LabVIEW-program (National Instruments). All cells were cultured as described above and harvested at a confluency of approximatively $80 \%$.

\subsection{Statistical evaluation}

Testing significant differences when comparing pairs of conditions or cell populations, the area of overlap between the probability density distributions of the fitted parameters was computed. 
Differences were considered statistically significant for an overlap percentage of $* p<0.05, * * \mathrm{p}$ $<0.005$, and $* * * \mathrm{p}<0.0005$ by either Student's ( $\mathrm{t}$ ) or Anova test.

\section{Results}

3.1. ERK1/2 signaling participates in the alteration of force-generating structures in muscle cells expressing mutated A-type lamins

To examine whether actin-containing cytoskeletal structures were affected in muscle cells expressing mutated lamin A, we examined actin stress fibers and focal adhesions $[29,30]$. We observed that both the number and size of focal adhesions (Fig. 1A+inset) as well as paxillin, vinculin, and p-FAK expression (Fig. 1B+plot) were reduced in mutated C2-H222P cells compared with C2-WT cells. Treating C2-WT and C2-H222P cells with cytochalasin D (Fig. 1C+inset) depolymerized the actin network and subsequently changed the focal adhesion complex structure and size; and washing-out these drugs led to the re-polymerization of F-actin. However, actin re-polymerization and focal adhesion complex formation were slower in $\mathrm{C} 2$ H222P cells compared with C2-WT cells. These effects were diminished when selumetinib, a selective inhibitor of MEK1/2, was added to the cell culture. In summary, these findings indicate that ERK1/2 signaling affects actin polymerization and focal adhesion formation in muscle cells expressing mutated lamin A.

In muscle cells, actin stress fibers interact with myosin II and are the force-generating structures that respond to the surrounding physical environment [31]. Looking at the stress fiber and myosin II distribution (Fig. 1D+inset) and the expression of myosin II in these cells (Fig. $1 \mathrm{E}+\mathrm{plot})$, we showed that they were significantly decreased in $\mathrm{C} 2-\mathrm{H} 222 \mathrm{P}$ cells compared with C2-WT cells but increased in $\mathrm{C} 2-\mathrm{H} 222 \mathrm{P}$ cells when treated with selumetinib. These data indicate that the $\mathrm{H} 222 \mathrm{P}$ lamin A variant disrupts the organization of stress fibers in muscle 
cells, which is under the control of ERK1/2 signaling.

\subsection{ERK1/2 signaling decreases cellular migration abitity caused by mutated A-type lamins}

Since mutated lamin A/C alters the intracellular actin cytoskeleton [24, 25, 32], we hypothesize that this could also impact cytoskeleton-based cellular processes, e.g. ERK1/2 signalingdependent actin dynamic processes such as cell locomotion. To investigate the effect of mutated lamin A on cell migration, C2-WT and $\mathrm{C} 2-\mathrm{H} 222 \mathrm{P}$ muscle cells were grown to confluency and scratched to create a wound. The rate of wound healing was then monitored over time. The results showed that wound closure of C2-H222P cells was slower compared with C2-WT cells (Fig. 2A). Adding selumetinib to $\mathrm{C} 2-\mathrm{H} 222 \mathrm{P}$ cells, the wound healing rate increased and was similar to C2-WT cells as shown in (Fig. 2B). This observation indicates that ERK1/2 signaling affects migration in muscle cells expressing mutated lamin A.

It has previously been reported that cortactin associates with actin and regulates the cytoskeletal network through several pro-migratory signaling pathways [33, 34]. Serine phosphorylation of cortactin is known to govern its ability to regulate actin organization [35, 36]. We therefore tested whether phosphorylated (=activated) cortactin was changed in C2-WT and C2-H222P cells. The expression of phosphorylated cortactin at position S405 and S418 by ERK1/2 signaling was previously described $[37,38]$. We found that $\mathrm{p}$-cortactin expression was increased at position (S418) and (S405) relative to total cortactin in C2-H222P cells compared to C2-WT cells (Fig. 3A); however, the phosphorylation was significantly reduced after the addition of selumetinib to C2-H222P cells. Further, the ratio of total cortactin to control protein (gapdh) and pERK1/2 to ERK1/2 expression was notably higher in C2-H222P cells compared to C2-WT and C2-H222P treated with selumetinib (Fig. 3B). Overall, these results indicate that pERK1/2 catalyzes the serine phosphorylation of cortactin in muscle cells expressing lamin A 
H222P, which correlates with altered F-actin dynamics (Fig. 1C) and decreased cellular movement (Fig. 2B).

\subsection{ERK1/2 signaling modulates cellular stiffness in muscle cells expressing mutated A-type} lamins

We imaged C2-WT and C2-H222P cells with a high-speed camera as they passed through the micro-constriction device and determined the mechanical properties from the apparent cell deformation, where $\varepsilon_{\max }$ equals the cell strain, $\Delta p$ the pressure drop, and $t_{\text {entry }}$ the cell passing time (Fig. 4A). The apparent cell mechanical response to micro-constrictions was determined by the effect of stress/strain stiffening [28].

The measured values, which are connected to a power-law relationship, allowed the determination of the stiffness of C2-WT cells and C2-H222P cells in the presence/absence of selumetinib. We found that the cell stiffness was increased in mutated cells compared to wild type cells (Fig. 4B). However, when selumetinib was added, the cell stiffness was reduced to wild type level, showing that ERK1/2 signaling caused changes in mechano-signaling in muscle cells expressing mutated lamin A.

To gauge whether the difference in cell stiffness was associated with increased remodeling dynamics of the cytoskeleton, we also measured the power-law exponent of the creep response (Fig. 4C). A higher power-law exponent indicates a more fluid-like behavior of the cell due to higher dynamics of cytoskeletal components $(39,40]$. The fluidity (i.e. cytoskeletal dynamics) was slightly decreased in C2-H222P compared to C2-WT cells (Fig. 4C), but similar between $\mathrm{C} 2-\mathrm{H} 222 \mathrm{P}$ and $\mathrm{C} 2-\mathrm{H} 222 \mathrm{P}$ cells plus selumetinib, indicating that mutated lamin A might stabilize cytoskeletal structures. However, treating C2-H222P cells with 
selumetinib led to a decrease in cellular stiffness compared to untreated cells (Fig. 4B), which may be associated to increases in focal adhesion protein and actomyosin expression in cells treated with the inhibitor by ERK1/2 signaling (Fig. 1B+E).

\section{Discussion}

Our findings that $L M N A$ mutation leads to changes in the cytokeletal network confirms work from others $[11,12]$. They suggested that LMNA mutations associated with striated muscle disorders render cells more sensitive to mechanical stress and mechanosignaling/transduction [13-15], and the aberrant activation of stress-activated ERK1/2 signaling might be the reason for it $[16,23]$.

It is known that A-type lamins connect the actin cytoskeleton through the (LINC) complex, which comprises of the nesprin and SUN 1/2 proteins. This molecular connection between the nuclear envelope and cytoskeletal elements is believed to serve as a mechanosensor, translating mechanical cues into biochemical signals, thus allowing cells to adapt to their physical environment [41, 42]. Kanellos et al. [43] showed that the decoupling of the nucleus and cytoskeleton by knocking-down nesprin-2 and SUN1 components of the LINC complex or ADF/cofilin-1 is responsible for promoting nuclear deformation, most likely by inverting increased contractile forces to the nuclear envelope via its attachments to the LINC complex. This implies that dynamic regulation of actin by ADF/cofilin-1 might be important for the actin-LINC complex to control nuclear shape.

In our study using micro-constriction rheology, we observed differences in the mechanics of C2-H222P cells expressing mutated lamin A and wild-type (C2-WT) cells. The high sensitivity of this method to cytoskeletal and nuclear changes in these cells was shown, when the chemical substance selumetinib was added to $\mathrm{C} 2-\mathrm{H} 222 \mathrm{P}$ cells. The mechanics of $\mathrm{C} 2-$ 
H222P cells was restored back to wild-type C2-WT level, where stress/strain stiffening determined cellular mechanics.

Based on all our studies, we therefore propose that in muscle cells expressing mutated

lamin A, the aberrant activation of ERK1/2 signaling leads to phosphorylation of cortactin (S405, S418), activates ADF/cofilin-1, alters actin dynamics as well as actin-containing structures, focal adhesion protein expression, and ultimately participates in cellular mechanics.

\section{Acknowledgements}

We thank Dr. Ben Fabry for expert advice, Dr. Scott A. Weed for the phosphorylated-cortactin (S405, S418) antibodies, and Celia Marshall (MA) for proofreading the manuscript. This work was supported by grants from the European Union Horizon 2020 program Phys2BioMed, No. 812772 (WHG) and Association Française contre les Myopathies from the Institut National de la Santé et de la Recherche Médicale and Sorbonne Université (AM).

\section{References}

1. Herrmann H, Bär H, Kreplak L, Strelkov SV, Aebi U, Intermediate filaments: from cell architecture to nanomechanics, Nat. Rev. Mol. Cell Biol. 7 (2007) 562-573.

2. Gregor M, Osmanagic-Myers S, Burgstaller G, Wolfram M, Fischer I, Walko G, Resch GP, Jorgl, Herrmann H, Wiche G, Mechanosensing through focal adhesion-anchored intermediate filaments, FASEB J. 28 (2014) 715-729.

3. Goldmann WH, Intermediate filaments and cellular mechanics, Cell Biol. Int. 39 (2018) 132-138.

4. Aebi U, Cohn J, Buhle L, Gerace L, The nuclear lamina is a meshwork of intermediate- 
type filaments, Nature 323 (1986) 560-564.

5. Turgay Y, Eibauer M, Goldman AE, Shimi T, Khayat M, Ben-Harush K, DubrovskyGaupp A, Sapra KT, Goldman RD, Medalia O, The molecular architecture of lamins in somatic cells, Nature 543 (2017) 261-264.

6. Lele TP, Dickinson RB, Gundersen GG, Mechanical principles of nuclear shaping and positioning, J. Cell Biol. 217 (2018) 3330-3342.

7. Chang W, Worman HJ, Gundersen GG, Accessorizing and anchoring the LINC complex for multifunctionality, J. Cell Biol. 208 (2015) 11-22.

8. Gundersen GG, Worman HJ, Nuclear positioning, Cell 152 (2013) 1376-1389.

9. Kirby TJ, Lammerding J, Emerging views of the nucleus as a cellular mechanosensor, Nat. Cell Biol. 20 (2018) 373-381.

10. Maniotis AJ, Chen CS, Ingber DE, Demonstration of mechanical connections between integrins, cytoskeletal filaments, and nucleoplasm that stabilize nuclear structure, Proc. Natl. Acad. Sci. USA 94 (1997) 849-854.

11. Davidson PM, Lammerding J, Broken nuclei - lamins, nuclear mechanics, and disease, Trends Cell Biol. 24 (2014) 247-56.

12. Hah J, Kim DH, Deciphering nuclear mechanobiology in laminopathy, Cells 8 (2019) 3.

13. Lammerding J, Fong LG, Ji JY, Reue K, Stewart CL, Young SG, Lee RT, Lamins A and C but not lamin B1 regulate nuclear mechanics, J. Biol. Chem. 281 (2006) 2576825780.

14. Swift J, Ivanovska IL, Buxboim A, Harada T, Dingal PC, Pinter J, Pajerowski JD, Spinler KR, Shin JW, Tewarai M, Rehfeldt F, Speicher DW, Discher DE, Nuclear lamin-A scales with tissue stiffness and enhances matrix-directed differentiation, Science 341 (2013) 1240104. 
15. Lin F, Worman HJ, Stuctural organization of the human gene encoding nucelar lamin A and nucelar lamin C, J. Biol. Chem. 168 (1993) 16321-16326.

16. Bonne G, Di Barletta MR, Varnous S, Bécane HM, Hammouda EH, Merlini L, Muntoni F, Greenbarg CR, Gary F, Urtizberea JA, Duboc D, Fardeau M, Toniolo D, Schwartz $\mathrm{K}$, Mutations in the gene encoding lamin A/C cause autosomal dominant EmeryDreifuss muscular dystrophy, Nat. Genet. 21 (1999) 285-288.

17. Emery AE, X-linked muscular dystrophy with early contractures and cardiomyopathy (Emery-Dreifuss type), Clin. Genet. 32 (1987) 360-367.

18. Muchir A, Bonne G, van der Kooi AJ, van Meegen M, Baas F, Bolhuis PA, de Visser M, Schwartz K, Identification of mutations in the gene encoding lamins $\mathrm{A} / \mathrm{C}$ in autosomal dominant limb girdle muscular dystrophy with atrioventricular conduction disturbances (LGMD1B), Hum. Mol. Genet. 9 (2000) 1453-1459.

19. Quijano-Roy S, Mbieleu B, Bönneman CG, Jeannet PY, Colomer J, Clarke NF, Cuisset JM, Roper H, De Meirleir L, D’Amico A, Ben Yaou R, Nascimento A, Barois A, Demay L, Bertini E, Ferreiro A, Sewry CA, Romero NR, Ryan M, Muntoni F, Guicheney P, Richard P, Bonne G, Estournet B, De novo LMNA mutations cause a new form of congenital muscular dystrophy, Ann. Neurol. 64 (2008) 177-186.

20. Fatkin D, MacRae C, Sasaki T, Wolff MR, Porcu M, Frenneaux M, Atherton J, Vidaillet HJ Jr, Spudich S, De Girolami U, Seidman JG, Seidman C, Muntoni F, Müehle G, Johnson W, McDonough B, Missense mutations in the rod domain of the lamin A/C gene as causes of dilated cardiomyopathy and conduction-system disease, N. Engl. J. Med. 341 (1999) 1715-1724.

21. Broers JL, Peeters EA, Kuijpers HJ, Endert J, Bouten CV, Oomens CW, Baaijens FP, Ramaekers FC, Decreased mechanical stiffness in LMNA-/- cells is caused by defective nucleo-cytoskeletal integrity: implications for the development of laminopathies, Hum. 
Mol. Genet. 13 (2004) 2567-2580.

22. Worman HJ, Bonne G, Laminopathies: a wide spectrum of human diseases, Exp. Cell Res. 313 (2007) 211-2133.

23. Muchir A, Pavlidis P, Decostre V, Herron AJ, Arimura T, Bonne G, Worman HJ, Activation of MAPK pathways links LMNA mutations to cardiomyopathy in EmeryDreifuss muscular dystrophy, J. Clin. Invest. 117 (2007) 1282-1293.

24. Chatzifrangkeskou M, Yadin D, Marais T, Chardonnet S, Cohen-Tannoudji M, Mougenot N, Schmitt A, Crasto S, Di Pasquale E, Macquart C, Tanguy Y, Jebeniani I, Puceat M, Morales Rodriguez B, Goldmann WH, Dal Ferro M, Biferi MG, Knaus P, Bonne G, Worman HJ, Muchir A, Cofilin-1 phosphorylation catalyzed by ERK1/2 alters cardiac actin dynamics in dilated cardiomyopathy caused by lamin A/C gene mutation, Hum. Mol. Genet. 27 (2018) 3060-3078.

25. Antoku S, Wu W, Joseph LC, Morrow JP, Worman HJ, Gundersen GG, ERK1/2 Phosphorylation of FHOD Connects Signaling and Nuclear Positioning Alternations in Cardiac Laminopathy, Dev. Cell 51 (2019) 602-616.e12.

26. Isermann $\mathrm{P}$, Lammerding $\mathrm{J}$, Nuclear mechanics and mechanotransduction in health and disease, Curr. Biol. 23 (2013) R1113-1121.

27. Choi JC, Wu W, Muchir A, Iwata S, Homma S, Worman HJ, Dual specificity phosphatase 4 mediates cardiomyopathy caused by lamin A/C (LMNA) gene mutation, J. Biol. Chem. 287 (2012) 40513-40524.

28. Lange JR, Metzner C, Richter S, Schneider W, Spermann M, Kolb T, Whyte G, Fabry B, Unbiased High-Precision Cell Mechanical Measurements with Micro-constrictions, Biophys. J. 112 (2017) 1472-1480.

29. Tojkander S, Gateva G, Lappalainen P, Actin stress fibers-assembly, dynamics and biological roles, J. Cell Sci. 125 (2012) 1855-1864. 
30. Burridge K, Focal adhesions: a personal perspective on a half century of progress, FEBS J. 284 (2017) 3355-3361.

31. Burridge K, Wittchen ES, The tension mounts: stress fibers as force-generating mechanotransducers, J. Cell Biol. 200 (2012) 9-19.

32. Ho CY, Jaalouk DE, Vartiainen MK, Lammerding J, Lamin A/C and emerin regulate MKL1-SRF activity by modulating actin dynamics, Nature 497 (2013) 507-511.

33. Daly RJ, Cortactin signalling and dynamic actin network, Biochem. J. 382 (2004) 1325.

34. Ammer AG, Weed SA. Cortactin branches out: roles in regulating protusive actin dynamics, Cell Motil. Cytoskeleton 65 (2008) 687-707.

35. Lua BL, Low BC, Cortactin phosphorylation as a switch for actin cytoskeletal network and cell dynamics control, FEBS Lett. 579 (2005) 577-585.

36. Kelley LC, Hayes KE, Ammer AG, Martin KH, Weed SA, Cortactin phosphorylated by ERK1/2 localizes to sites of dynamic actin regulation and is required for carcinoma lamellipodia persistence, PLoS One 5 (2010) e13847.

37. Martinez-Quiles N, Ho HY, Kirschner MW, Ramesh N, Geha RS, Erk/Src phosphorylation of cortactin acts as a switch on-switch off mechanism that controls its ability to activate N-WASP, Mol. Cell Biol. 24 (2004) 5269-5280.

38. Kruchten AE, Krüger EW, Wang Y, McNiven MA, Distinct phspho-forms of cortactin differentially regulate actin polynerization and focal adhesions, Am. J. Physiol. Cell Physiol. 295 (2008) C1113-1122.

39. Bursac P, Lenormand G, Fabry B, Oliver M, Weitz DA, Viasnoff V, Butler JP, Fredberg JJ, Cytoskeletal remodelling and slow dynamics in the living cell, Nat. Mater. 4 (2005) $557-561$.

40. Lange JR, Goldmann WH, Alonso JL, Influence of $\alpha v \beta 3$ integrin on the mechanical 
properties and the morphology of M21 and K562 cells, Biochem. Biophys. Res. Comm. 478 (2016) 1280-1285.

41. Crisp M, Liu Q, Roux K, Rattner JB, Shanahan C, Burke B, Stahl PD, Hodzic D, Coupling of the nucleus and cytoplasm: role of the LINC complex, J. Cell Biol. 172 (2006) 41-53.

42. Starr DA, Fridolfsson HN, Interactions between nuclei and the cytoskeleton are mediated by SUN-KASH nuclear-envelope bridges, Annu. Rev. Cell. Dev. Biol. 26 (2010) 421-444.

43. Kanellos G, Zhou J, Patel H, Rigway RA, Huels D, Gumiak CB, Sandilands E, Carragher NO, Sansom OJ, Witke W, Brunton VG, Frame MC, ADF and cofilin1 control actin stress fibers, nuclear integrity, and cell survival, Cell Rep. 13 (2015) 1-16.

\section{Figures and legends}

Fig. 1. ERK1/2 signaling changes focal adhesions in muscle cells expressing mutated lamin A.

(A) Representative immuofluorescence micrographs of focal adhesion staining of C2-

WT and C2-H222P cells and nuclei counter-stained with DAPI. (B) Immunoblots of phosphorylated-FAK, paxilin, and vinculin expression from C2-WT and C2-H222P cells with relative protein expression plots compared to ERK1/2 by Student's t-test. (C) Micrographs of $\mathrm{C} 2-\mathrm{WT}, \mathrm{C} 2-\mathrm{H} 222 \mathrm{P}$, and $\mathrm{C} 2-\mathrm{H} 222 \mathrm{P}$ cells treated with $50 \mu \mathrm{M}$ selumetinib for $15 \mathrm{~h}$ show phalloidin (F-actin) and paxilin staining during the cytochalasin D washout experiment. Micrographs were taken 5 min and 30 min after washout. Insets show representative areas. (D) Representative immuofluorescence micrographs by stress fiber staining of C2-WT and C2-H222P cells as well as nuclei counterstained with DAPI. (E) Immunoblots show non-muscle myosin II expression of 
C2-WT, C2-H222P, and C2-H222P cells treated with $50 \mu \mathrm{M}$ selumetinib for $15 \mathrm{~h}$ and a MII/ERK1/2 protein expression plot analyzed by Student's t-test $\left(* \mathrm{p}<0.05\right.$ and $\mathrm{p}^{* *}<$ 0.005). ERK1/2 was used as loading control. In summary, selumetinib may affect ERK1/2 signaling, actin polymerization, focal adhesion formation, and myosin II expression.

Fig. 2. Wound closure of muscle cells expressing mutated lamin A. (A) Cellular wound closure was analyzed with C2-WT, C2-H222P, and C2-H222P cells treated with $50 \mu \mathrm{M}$ selumetinib after $0,3,6,9$, and $12 \mathrm{~h}$ post-wounding. (B) A plot (\% open wound) over time using an Anova test $(* * * p<0.0005)$. In summary, selumetinib may trigger ERK1/2 signaling and cellular migration in $\mathrm{C} 2-\mathrm{H} 222 \mathrm{P}$ cells.

Fig. 3. ERK1/2 drives the serine phosphorylation of cortactin in muscle cells expressing mutated lamin A. (A) The immunoblot shows phosphorylated (pS418) and (pS405) as well as total cortactin and phosphorylated and total ERK1/2 in C2-WT, C2-H222P, and C2-H222P cells treated with $50 \mu \mathrm{M}$ selumetinib with gapdh as loading control. (B) Relative protein expression plots using an Anova test $\left(* \mathrm{p}<0.05\right.$ and $\left.\mathrm{p}^{* * *}<0.0005\right)$.

Fig. 4. Mechanics of C2-WT, C2-H222P, and C2-H222P cells treated with $50 \mu \mathrm{M}$ selumetinib using micro-constriction rheology. (A) In each plot, only cells that experienced the same deformation and pressure during the measurements were selected for quantitative comparison. (B) Cell stiffness and (C) Cell fluidity [28]. Error bars represent S.E. and were calculated by bootstrapping. $* \mathrm{p}<0.05$ (Student t-test). In summary, selumetinib may stimulate ERK1/2 signaling in C2-H222P cells by destabilizing the nucleus and cytoskeleton and increasing cellular compliance. 
A.
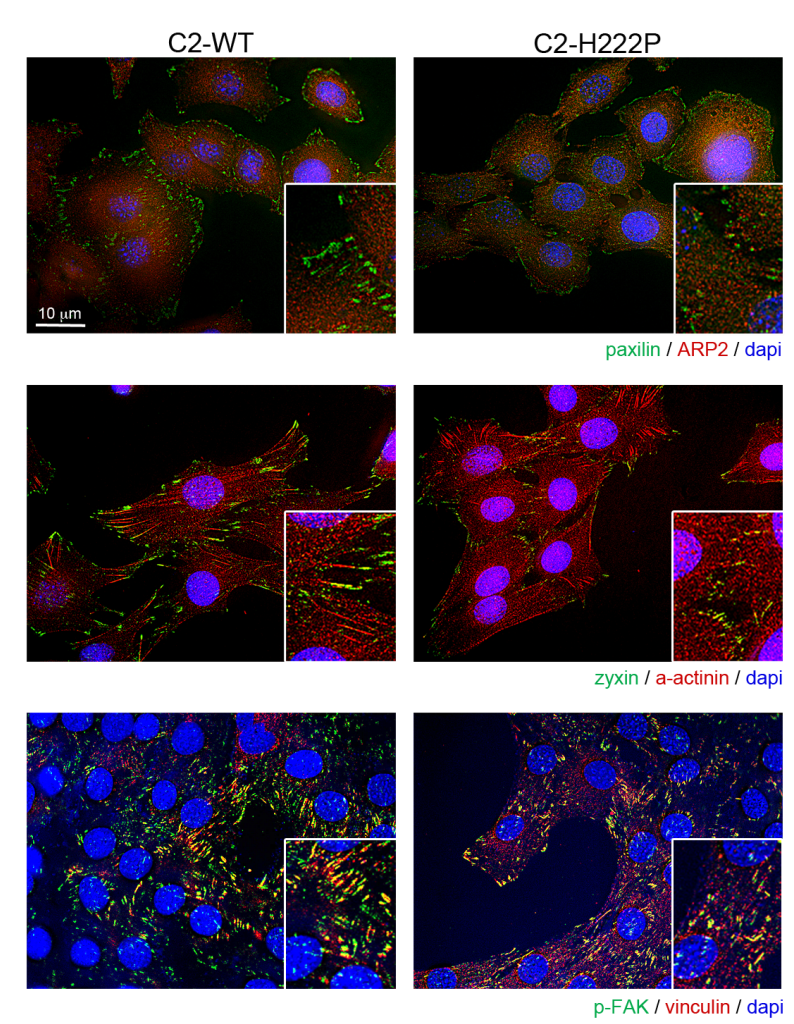

B.

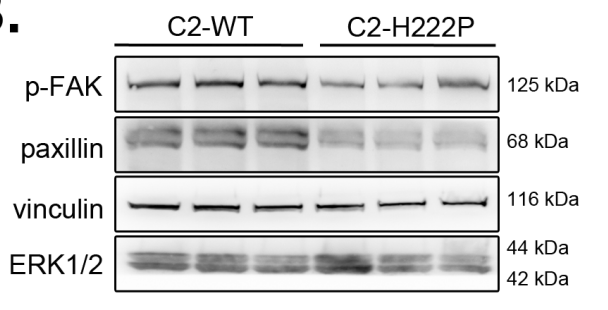

C.

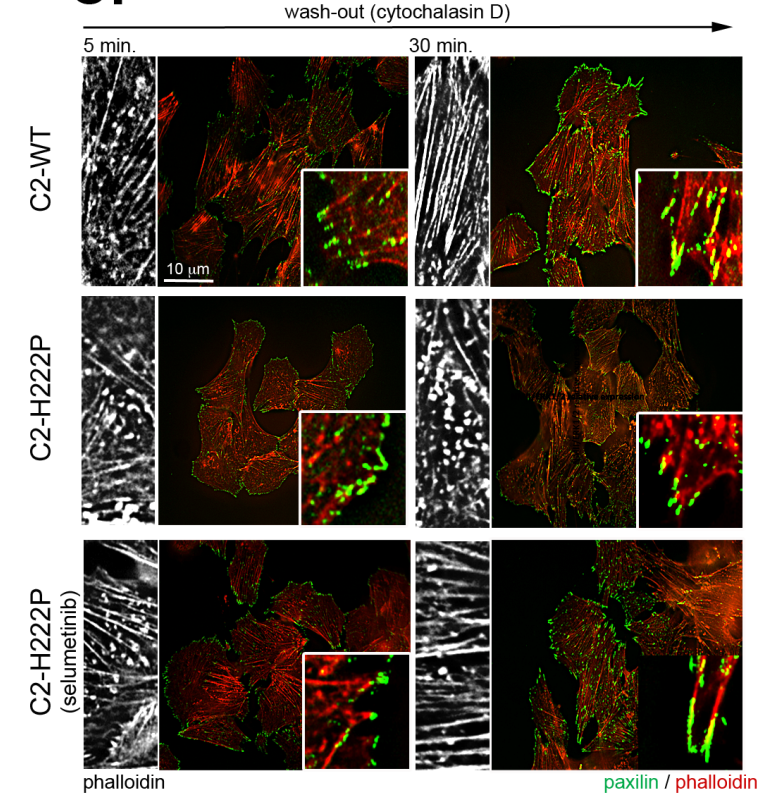

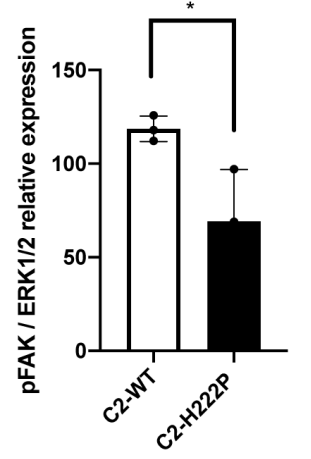
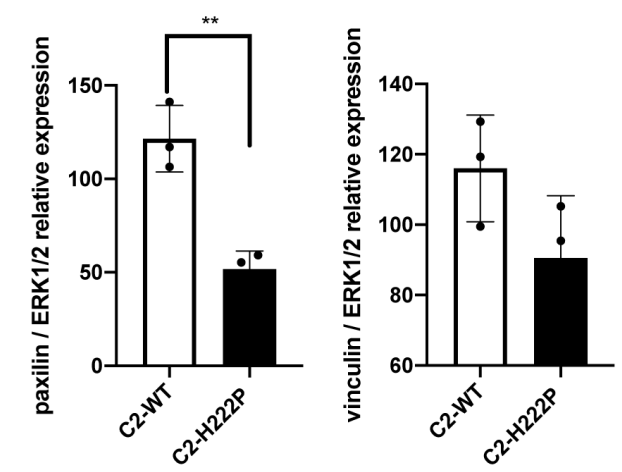

D.

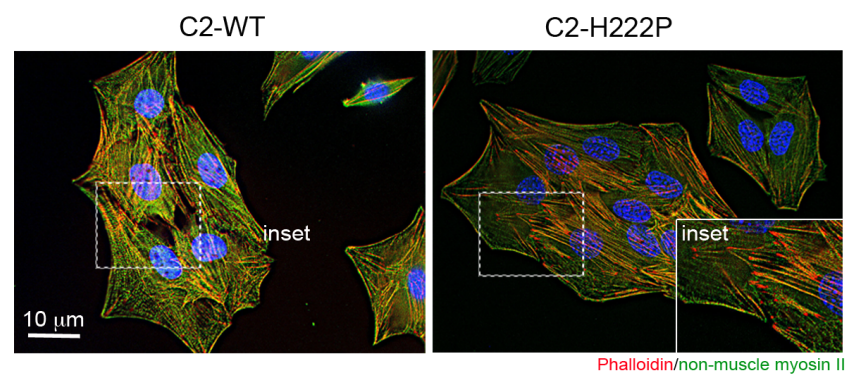

E.

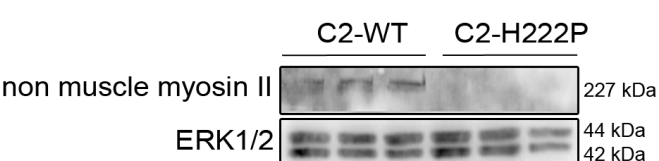
$\frac{\mathrm{C} 2-\mathrm{H} 222 \mathrm{P}}{-}$ selumetinit

non muscle myosin 11 $\operatorname{ERK} 1 / 2====\equiv=\begin{aligned} & 44 \mathrm{kDa} \\ & 42 \mathrm{KDa}\end{aligned}$

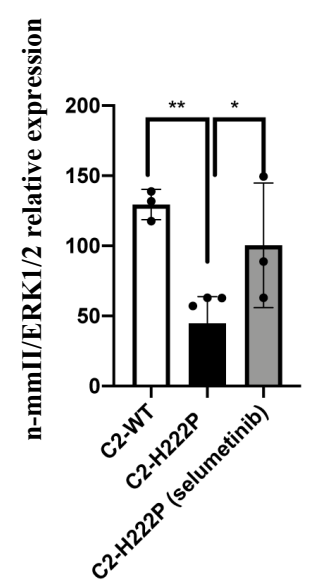


A.

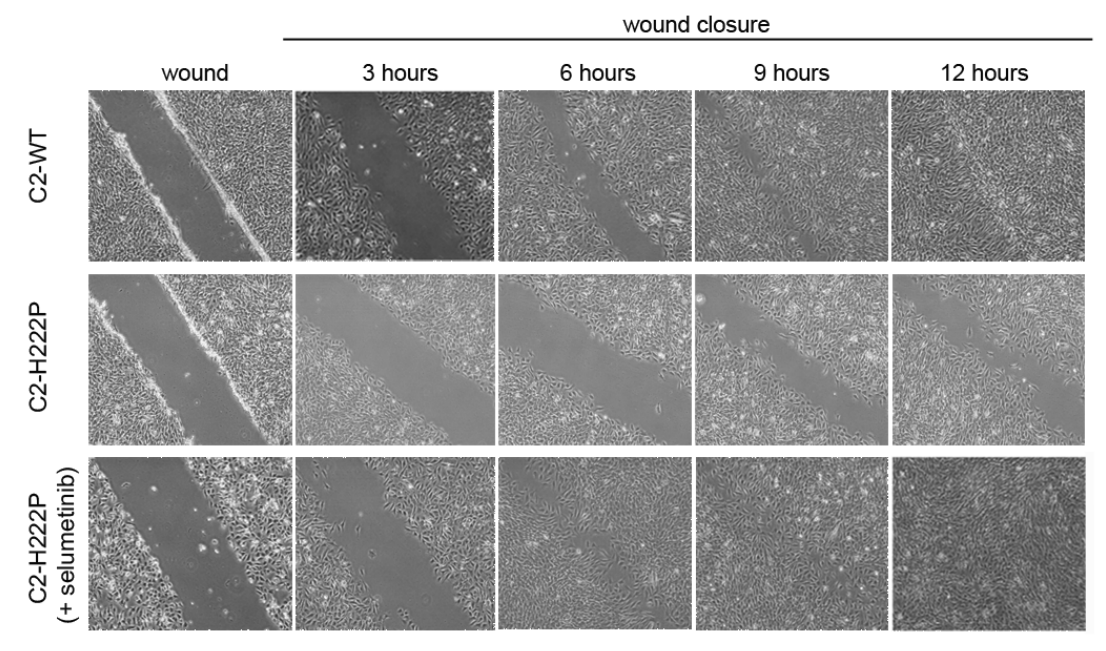

B.

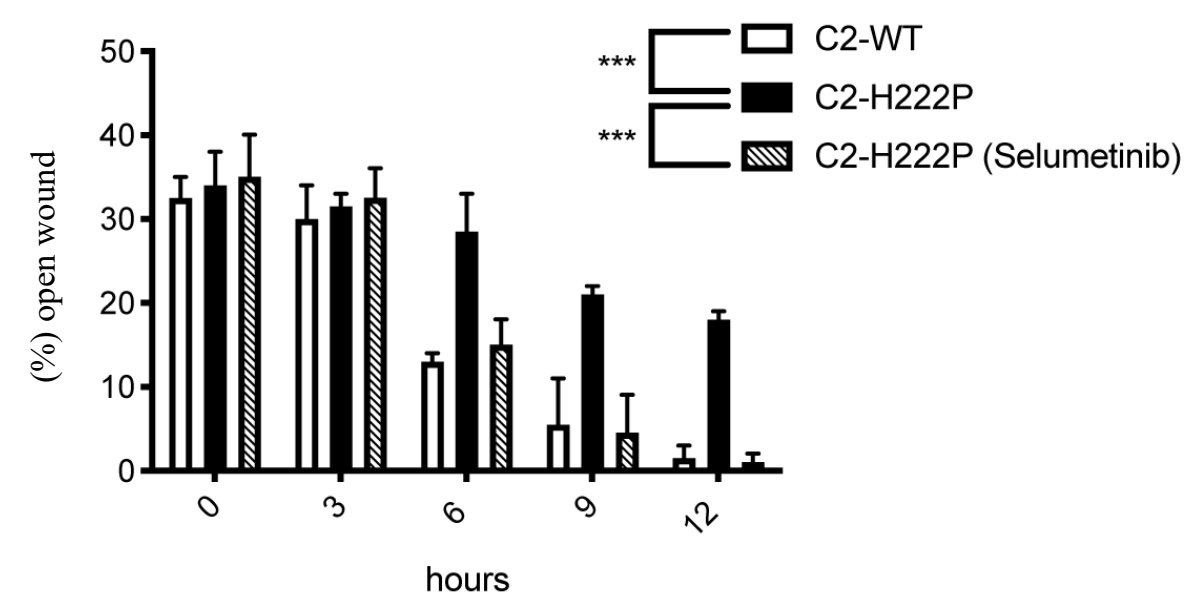


Figure 3

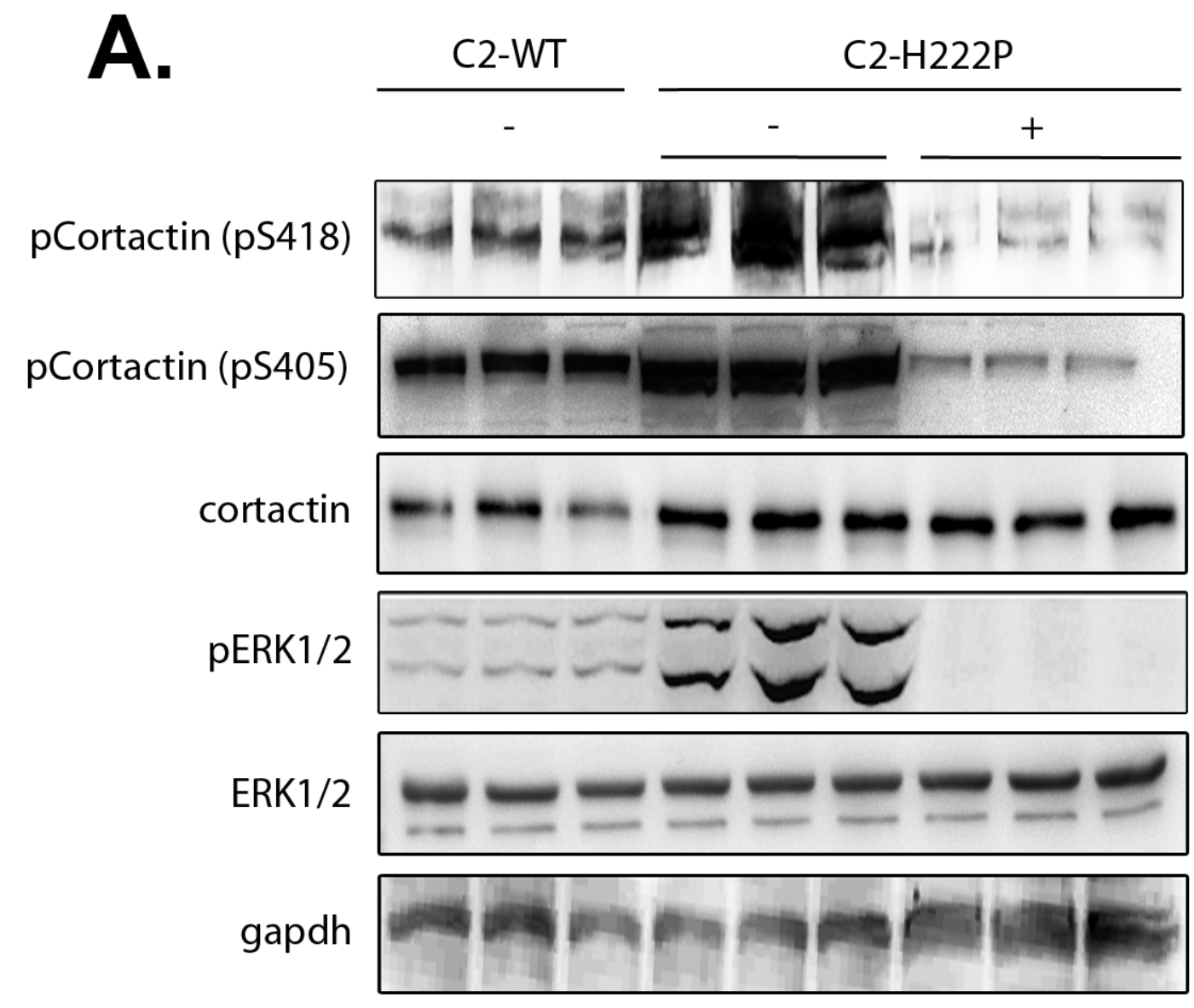

B.
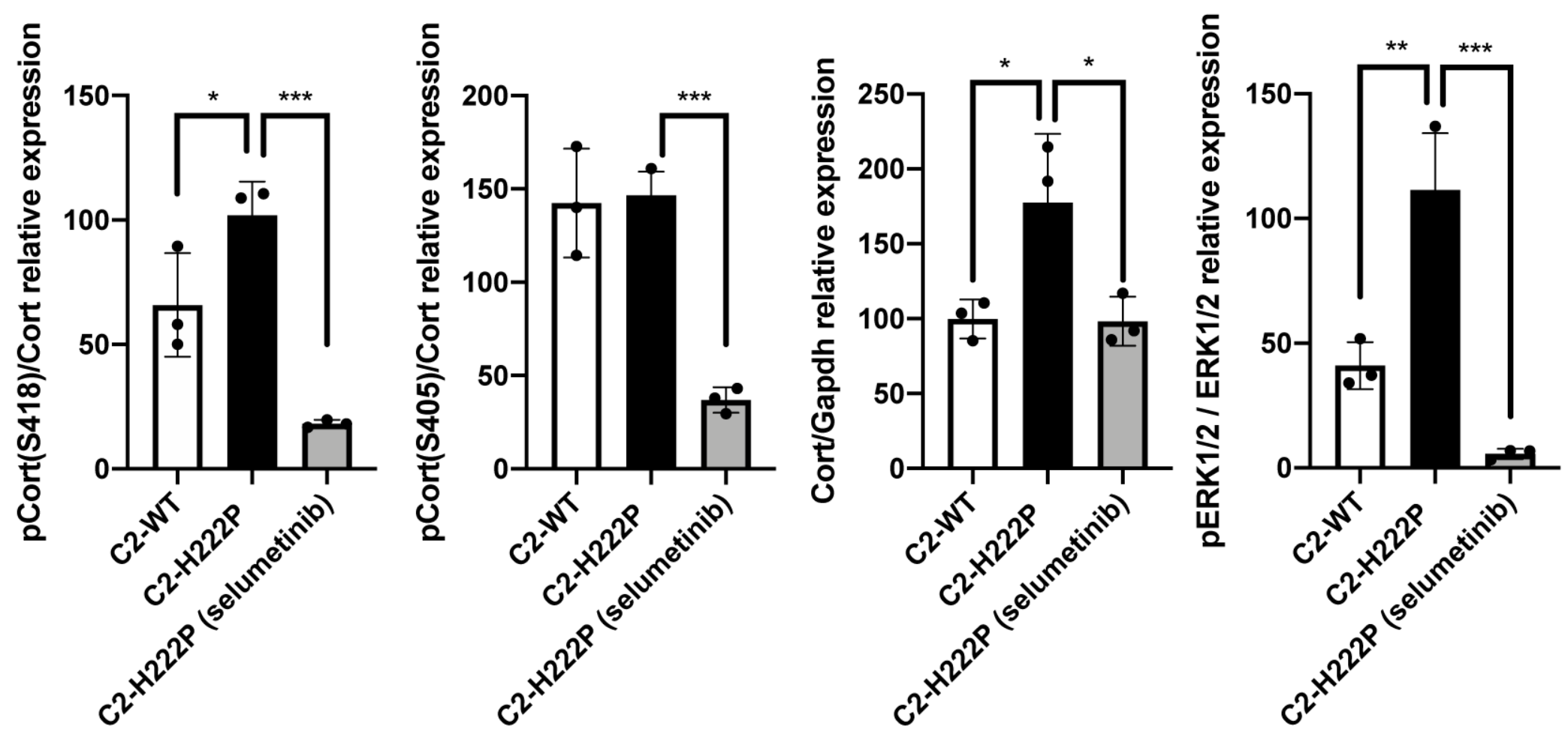

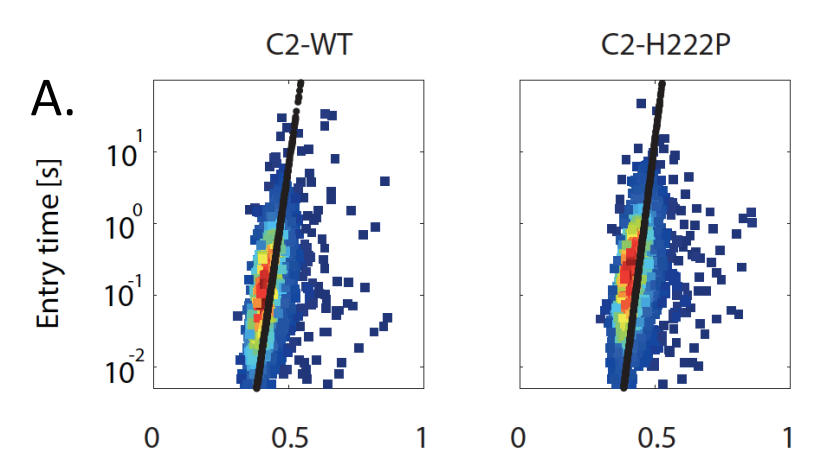

$\varepsilon / \Delta p$ (Creep modulus) $[1 / \mathrm{Pa}$
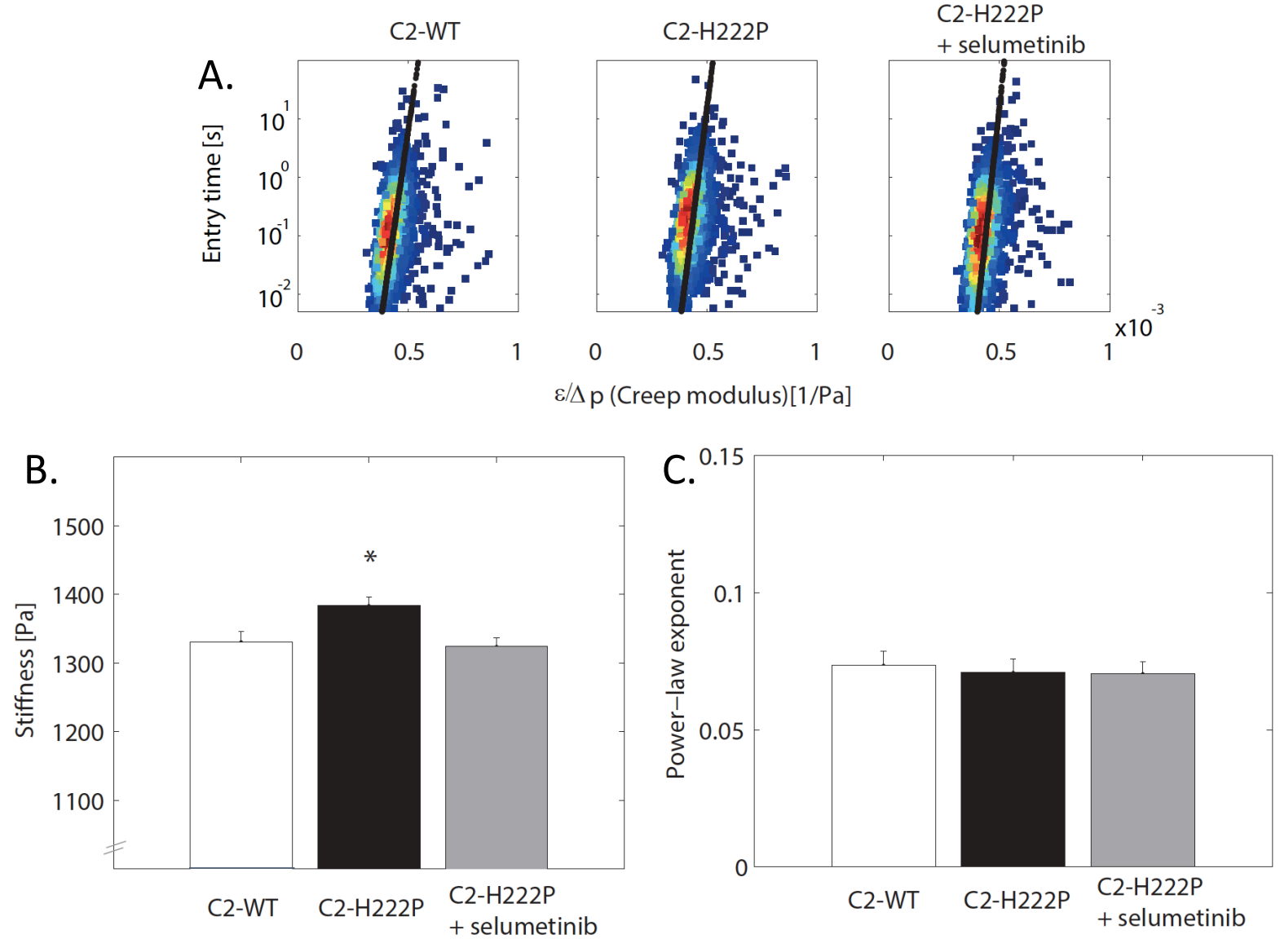
This is to confirm that the publication of this manuscript does not conflict with any financial or any other interest. 